\title{
TAVOLA PERIODICA SOTTO PRESSIONE: UNA PERIODICITÀ DIVERSA ED UNA CHIMICA ESOTICA
}

\author{
CARLO EDOARDO GATTI (*)
}

\begin{abstract}
SunTO. - La chimica, così come la conosciamo e come l'utilizziamo per descrivere ed interpretare le proprietà strutturali, di legame, di reattività dei diversi composti, si basa essenzialmente sulla periodicità delle proprietà degli atomi costituenti. Questa periodicità ha ispirato la proposizione della Tavola Periodica degli elementi e ha poi guidato la sua razionalizzazione, grazie alle conoscenze via via acquisite sulla struttura elettronica atomica. La periodicità dei raggi atomici riflette la periodicità delle configurazioni elettroniche del guscio atomico più esterno. La diversità dei raggi differenzia le proprietà chimiche e di conseguenza le strutture e proprietà dei solidi elementari e dei loro composti. Ma sotto una pressione esterna lo scenario può cambiare anche drasticamente. Quando un atomo viene compresso, la sua densità elettronica media aumenta: poiché il guscio esterno è il più facilmente comprimibile, già a 100 Giga Pascal $(\mathrm{GPa})$, ovvero a $10^{6}$ atmosfere, la variazione di raggio atomico diventa molto meno marcata lungo la Tavola Periodica. A $1000 \mathrm{GPa}$ la nozione di periodicità è definitivamente persa. Sotto pressione, all'energia interna del sistema si somma l'energia dovuta al lavoro di compressione e con questa energia addizionale il sistema può esplorare regioni di energia potenziale normalmente inaccessibili. La natura del legame chimico cambia, anche drasticamente, e strutture e legami del tutto nuovi, caratterizzati da proprietà spesso inaspettate, diventano energeticamente stabili e dunque possibili. Ad esempio il Sodio, da metallo di colore argenteo, diventa totalmente trasparente ed isolante; il Boro diventa parzialmente ionico con trasferimento di carica tra gli atomi, in una fase cristallina elementare; l'inerzia chimica dell'Elio viene superata formando un composto con il sodio, $\mathrm{Na}_{2} \mathrm{He}$, altamente stabile. Si osservano composti con formule brute improbabili $\left(\mathrm{Na}_{3} \mathrm{Cl}_{2}, \mathrm{Na}_{2} \mathrm{Cl}, \mathrm{Na} \mathrm{Cl}_{3} \mathrm{NaCl}_{3}, \mathrm{NaCl}_{7}\right)$, si formano nuovi allotropi del carbonio, scompare l'aromaticità del benzene... Questa nuova chimica viene in genere predetta a livello computazionale e interpretata e razionalizzata con gli strumenti della meccanica quantistica e delle teorie più avanzate del legame chimico. Ma in molti casi i composti
\end{abstract}

(*) CNR-ISTM, Istituto di Scienze e Tecnologie Molecolari, Milano, Istituto Lombardo Accademia di Scienze e Lettere, Milano, Italia.

E-mail: c.gatti@istm.cnr.it 
predetti in silico si sono poi potuti riprodurre in laboratorio, utilizzando le celle ad incudine di diamante, e caratterizzare con varie analisi strumentali in situ.

$* * *$

ABSTRACT. - Our general and operative notion of chemistry, that enabling us to describe and interpret the structural, bonding and reactivity properties of the various compounds, is essentially rooted in the periodicity of properties of their constituting elements. Periodicity drove the path towards the proposition of the periodic table of elements and its rationalization thanks to the gradually acquired knowledge on the atomic electronic structure. Atomic radii periodicity mirrors the periodicity of the electronic configurations of the atoms' outermost shell. The changes in atomic radii differentiate the chemical properties, hence the structures and properties of the elemental solids and of their compounds. Yet, under an external pressure, such a fully rationalized scenario may drastically change. When an atom is compressed, its average electron density increases and its outermost electronic shell is the easiest one to compress. At 100 Giga Pascal $(\mathrm{GPa})$, that is at $10^{6}$ atmospheres, the change of the atomic radius along a period of the Periodic Table becomes much less evident and at $1000 \mathrm{GPa}$ our classical notion of periodicity is completely lost. Under pressure, the energy due to the compression work made on a system adds up to its internal energy. With such an energy gain the system may reach regions of the potential energy surface which would not be otherwise accessible. The chemical bond nature may change, even radically, and new structures and bonding patterns, characterized by totally unexpected properties, become energetically stable and possible. For instance, sodium, which is a silvery-white, highly reactive metal, becomes a fully transparent insulator, while boron is turned into a partially ionic solid elemental phase because charge transfer takes place between differently clustered groups of boron atoms. The incredible chemical inertness of helium finally falls as it forms a stable compound with sodium, $\mathrm{Na}_{2} \mathrm{He}$. Under a suitable pressure, compounds with unusual stoichiometry $\left(\mathrm{Na}_{3} \mathrm{Cl}_{2}, \mathrm{Na}_{2} \mathrm{Cl}, \mathrm{Na}_{3} \mathrm{Cl}, \mathrm{NaCl}_{3}, \mathrm{NaCl}_{7}\right)$ may be observed, despite their formula would be immediately rejected if proposed by a student at any high-school or university exam, or new carbon allotropes may appear or the aromatic character of benzene may vanish. This new chemistry is usually predicted through ab-initio quantum mechanical methods and interpreted and rationalized with the most modern chemical bonding approaches. However, compounds anticipated in silico have then be reproduced experimentally in many cases, by using diamond anvil cells to synthesize them and a variety of in situ instrumental techniques to characterize them properly.

\section{UN CAMBIO DI PROSPETTIVA: DAL SOGNO RIVELATORE DI MENDELEEV AD UN POSSIBILE INCUBO}

Dimitri Ivanovic Mendeleev era un valente chimico e, come molti altri chimici del suo tempo, coltivava il grande desiderio di ordinare gli elementi allora conosciuti, sessantatré nel 1869, secondo un qualche schema logico. È ben noto che fosse altresì un bravo giocatore di carte e soprattutto un vero appassionato di solitari. 
Tra leggenda e realtà [1-2], si racconta che avesse preparato dei cartoncini, uno per ogni elemento, con indicato simbolo e peso atomico, ${ }^{1} \mathrm{e}$ che si fosse poi messo a giocare con quei cartoncini, ordinandoli e organizzandoli come era avvezzo fare nei solitari... Ma quel solitario chimico non sembrava andare mai a buon fine. Dopo tre giorni e tre notti di tentativi frenetici, seduto al suo tavolo di lavoro, pare che se ne fosse andato a dormire esausto e frustrato. Come avviene sovente agli scienziati migliori, il suo cervello continuò nella notte a lavorare, sicuramente in modo più libero e meno preconcetto, così che in sogno gli permise finalmente di "vedere" quella disposizione, quella Tavola che stava appassionatamente cercando. Riportata poi su carta, ne conserviamo varie versioni, sia manoscritte, sia pubblicate su diverse riviste russe e tedesche. In una pubblicazione russa del 1869 [3] gli elementi di un gruppo sono disposti per riga (e quindi i periodi in colonna), ma in una successiva del 1871 [4] appaiono già allineati per colonna, come nella forma attuale più consueta della Tavola Periodica (TP). Disposti per riga o per colonna, gli elementi dei gruppi presentavano periodicità di proprietà così evidenti che Mendeleev si azzardò a inserire delle caselle vuote, assegnate ad elementi non ancora scoperti ma le cui proprietà erano state da lui correttamente ipotizzate, sulla base di quelle degli elementi tra i quali erano inseriti. [4] Di fatto, questi elementi vennero poi scoperti, il Gallio nel 1875, lo Scandio nel 1879, il Germanio nel 1886 ed il Tecnezio, elemento artificiale, nel secolo successivo, precisamente nel 1937 ad opera di due fisici italiani, Carlo Perrier ed Emilio Segrè, che lavoravano a Palermo nei laboratori dell'Istituto di Fisica. Naturalmente dei nuovi elementi vennero valutati anche i pesi atomici, che risultarono differire di poco da quelli previsti da Mendeleev. [4] Questa capacità di predizione è una splendida conferma della razionalità della disposizione degli elementi nella TP e del grado di informazione chimica contenuto nella nozione di periodicità. Ma se Mendeleev si fosse addestrato alla chimica, avesse giocato ai suoi solitari o perfino si fosse trovato a sognare sotto un'enorme pressione, all'interno di una gigantesca pressa o all'interno della terra, schiacciato dagli strati soprastanti, il suo sogno si sarebbe probabilmente trasformato in un

1 Riportò i pesi e non i numeri atomici $Z$ perché a quei tempi non si conosceva ancora nulla della struttura quantistica dell'atomo che sarebbe stata poi scoperta agli inizi del secolo successivo (si legga a questo proposito il testo della conferenza del Prof. Carlo Maria Prosperi). 
incubo...Tutte le periodicità che aveva mirabilmente disvelato e razionalizzato, sarebbero apparse, come vedremo, molto più indefinite ed il suo solitario non avrebbe mai avuto una risoluzione onirica così efficace.

\section{UNA NUOVA CHIMICA}

Mentre preparavo questa breve esposizione, mi sono chiesto se il titolo proposto non potesse suonare un po' irriverente rispetto alla celebrazione del $150^{\circ}$ anniversario di una scoperta che è stata ragionevolmente considerata come una vera rivoluzione copernicana per la chimica da J. D. Bernal, eminente storico della scienza, fisico e cristallografo. In effetti, curiosando in rete, non ho incontrato presentazioni analoghe, fra le molte proposte per questa speciale ricorrenza. Ma ho comunque potuto presto tranquillizzarmi, quando ho trovato contributi molto autorevoli sul tema che avevo scelto. Desidero innanzitutto citare due scritti che mi sono stati di grande ausilio: La materia in condizioni estreme: una chimica dell'altro mondo [5], redatto dal Prof. Vincenzo Schettino, socio corrispondente dell'Accademia Nazionale dei Lincei e l'articolo di rassegna, The chemical imagination at work in very tight places, [6] scritto, tra gli altri, dal premio Nobel per la Chimica 1981, Roald Hoffman, e da Neil W. Ashcroft, autore insieme a N. David Mermin di uno dei più noti testi di fisica dello stato solido [7]. Richiamo inoltre un articolo, Pressure turns simplicity into complexity, [8] apparso su ESRFnews, la rivista che informa su quanto "bolle in pentola" all' European Synchrotron Radiation Facility (ESRF) di Grenoble. Presso questo laboratorio si utilizza da anni la radiazione di sincrotrone $X$ anche per studiare le variazioni strutturali sotto pressione, conducendo esperimenti che "are rewriting the phase diagrams of the elements, revealing surprising complexity and new material behaviour". [8] Non è quindi un caso che uno dei sottoparagrafi dell'articolo sia profeticamente intitolato: The new Periodic Table. [8] Di fatto, malgrado siano state proposte più di ottocento forme della TP, di cui alcune molto curiose, non ne è stata ancora proposta una che descriva gli elementi chimici sotto pressione. Come ci sarà più chiaro in seguito, sarebbe probabilmente troppo complessa e naturalmente variabile, essa stessa, in funzione della pressione (e, in misura decisamente minore, anche della temperatura). Tutte le Tavole che sono state finora proposte, anche le meno convenzionali, sottolineano e presuppongono la periodicità delle proprietà degli elementi: questo vale anche per quella redatta, 
in occasione del $150^{\circ}$ anniversario, dalla European Chemical Society, nella quale gli elementi, sempre disposti periodicamente, sono però rappresentati da caselle più o meno grandi a seconda della loro abbondanza.[9] La pubblicazione di questa Tavola, particolarmente meritoria, è finalizzata a sensibilizzare ad un uso particolarmente morigerato di alcuni elementi, la cui disponibilità potrebbe mancare a breve, qualora venisse mantenuto lo standard attuale di impiego.

Che una possibile nuova chimica sia alle porte è anche evidenziato dalla divulgazione scientifica meno specialistica. Molto interessante è l'analisi riportata su New Scientist, una fra le testate più significative in tale settore, in un articolo intitolato Modern-day alchemy is putting the periodic table under pressure [10] e promosso sulla pagina di copertina della rivista con un titolo di forte impatto, The other Periodic Table, ed un sottotitolo che non è certo meno sconvolgente: The Chemistry we see isn't even the half of it. Ovvero, se da un lato la chimica che conosciamo non sarebbe che la punta di un iceberg, dall'altro il chimico moderno realizza tramite la pressione il sogno dell'alchimista di trasmutare gli elementi. In effetti sotto pressione, come vedremo, alcuni elementi si comportano come il successivo del loro gruppo a pressioni ordinarie. La Fig. 1 mostra questo alchimista moderno all'opera e ci fa intuire che la sua capacità di modificare le proprietà degli elementi e della materia attraverso la pressione nasce da una modifica delle loro orbite elettroniche, specialmente le più esterne, ovvero da una riduzione del volume di spazio a loro disposizione, con conseguenze che si ripercuotono anche su quelle più interne.

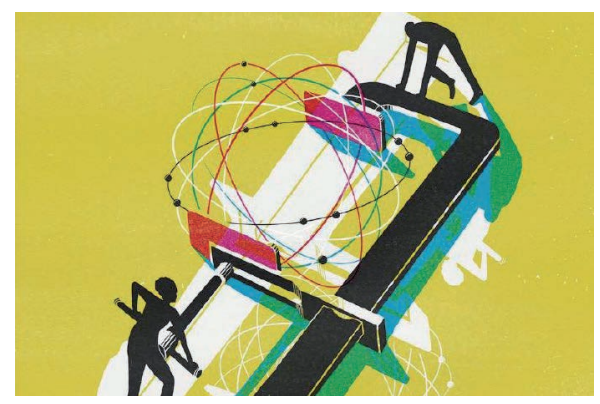

Fig. 1. L'alchimia dei giorni nostri mette la Tavola Periodica sotto pressione. La diminuzione del volume disponibile per le orbite elettroniche più esterne degli atomi di un sistema può modificarne in modo drastico le proprietà. Disegno di Daniel Stolle, [10] riprodotto con permesso da New Scientist, settembre 2015, copyright New Scientist Ltd. 
Ma prima di addentrarci sulla relazione tra pressione e struttura elettronica, facciamoci guidare in questa nuova avventura chimica da alcune delle asserzioni che Matthew Chalmers ha riportato nel suo articolo su New Scientist. [10] Le trascrivo in lingua originale perché particolarmente icastiche e le inframmezzo con qualche mia frase di raccordo o di commento per una più facile comprensione di un testo che non viene citato integralmente. La TP, così come la conosciamo "it is chemistry's poster child". Ovvero noi chimici ordinari, che lavoriamo a pressioni atmosferiche, non saremmo che dei bambini che si divertono con la scatola del "piccolo chimico", che ci permette di operare in condizioni note, senza sorprese e pericoli di imbatterci in qualcosa di poco comprensibile, se non pericoloso! "From copper's conductivity to mercury's mercurial liquidity, the periodic table assigns the chemical elements to neat columns and rows and so reveals their properties. It is chemists' first reference point in all their endeavours, whether building better catalytic converters, making concrete set faster or looking for the best materials for medical implants. Yet, this iconic picture of science is hopelessly parochial". Classificare la visione attuale della chimica come parrocchiale non ha nulla di dispregiativo, ma, come una parrocchia, malgrado la sua splendida dignità, non è che una piccola e limitata finestra sulla Chiesa Cattolica universale, così lo è la chimica dei nostri laboratori a pressioni ordinarie rispetto a ciò che avviene nel nostro universo. Di fatto, "most of the known matter in the universe doesn't exist under the cool, calm conditions of Earth's surface that the Periodic Table assumes. By mass, more than 99.9 per cent of normal matter resides within planets and stars - environments of high temperatures, but above all unimaginable pressures. Here, the elements' familiar identities start to blur...". Tuttavia, di questo mondo ad alta pressione conosciamo per ora pochissimo, "proportionally as if, in thousands of years of exploration, geographers had mapped out a region no larger than Spain... Slowly, however, modern-day alchemists are ratcheting-up the pressure. As they do, they are transforming the familiar physical and chemical properties of elements from $\mathrm{H}$ to $\mathrm{Fe}$, turning liquids to solids, non-metals to metals and more besides". E tutto ciò può avere anche un enorme interesse applicativo. In effetti, "...the aim is not just to understand more about the deep chemistry of our planet and others, but also to find materials that react more efficiently, store energy more effectively... Harnessing this extreme chemistry could yield astonishing new technologies". In pratica, come per tutte le imprese scientifiche di grande rilevanza e 
prospettiva, l'aspetto di conoscenza fondamentale si accompagna spesso ad un enorme potenziale applicativo.

\section{Pressione E SUOI INTERVALLI NELL'UNIVERSO}

Come è ben noto, la pressione P è la forza esercitata su una superficie e, a parità di forza, è inversamente proporzionale alla superficie su cui si applica la forza. Nel Sistema Internazionale, la sua unità di misura è il Pascal, pari alla forza di 1 Newton esercitata su 1 metro quadrato di superficie. Come vedremo meglio in seguito, quando un materiale è sottoposto a pressioni dell'ordine di 1-100 GigaPascal (GPa), ovvero 10'$10^{11} \mathrm{~Pa}$, si comincia ad osservare una chimica sorprendente, ben diversa da quella nota in condizioni normali (c.n.). Queste pressioni possono apparire del tutto inusuali se rapportate a quelle che regolano la vita sulla terra, cioè 1 atmosfera a livello del mare, pari a circa $10^{5} \mathrm{~Pa}$, e se si tiene conto che piccoli discostamenti del $1-2 \%$ da questo valore provocano effetti già significativi e spesso anche fastidiosi, a livello meteorologico e fisiologico, soprattutto per chi tra noi fosse più meteoropatico. Ma se ampliamo il nostro orizzonte di esplorazione, osserviamo che $\mathrm{P}$, una delle variabili termodinamiche fondamentali, ha nell'universo un'ampiezza di variazione di 64 ordini di grandezza, da $10^{-32}$ atmosfere negli spazi interstellari estremamente rarefatti a $10^{32}$ atmosfere al centro di una stella a neutroni.[5] Venendo più vicini alla nostra esperienza, la $\mathrm{P}$ su Marte, il pianeta che ci accingiamo ad esplorare a fondo con la missione ExoMars dell'Agenzia Spaziale Europea, è pari a 1/100 di quella terrestre, ma già in fondo alla fossa delle Marianne si raggiunge una $\mathrm{P}$ pari a $0.1 \mathrm{GPa}$, ovvero 1000 volte la $\mathrm{P}$ atmosferica. La produzione sintetica dei diamanti richiede pressioni dell'ordine dei $10 \mathrm{GPa}$, ovvero pressioni 100 volte maggiori e se scendiamo verso il centro del nostro pianeta la $\mathrm{P}$ aumenta in modo continuo fino a raggiungere pressioni dell'ordine dei 325-400 GPa nel nucleo interno della terra.

\section{LAVORARE AD ALTE PRESSIONI}

\subsection{L'alta pressione nella storia}

Il grande sviluppo degli studi ad alta pressione è fenomeno relati- 
vamente recente, ma, come ci ricorda Vincenzo Schettino, "una percezione pratica e intuitiva che l'applicazione di una forza e di una pressione potessero modificare in maniera sostanziale le proprietà della materia risale alla notte dei tempi e alle prime pratiche della metallurgia". [5] Ad esempio, nel sito di Cayonu Tepesi, in Anatolia centrale, sono stati rinvenuti circa 50 oggetti di rame, risalenti al VII-VIII millennio a.C., induriti per martellazione, e soggetti ad una parziale ricottura. La martellazione permetteva di raddoppiarne la durezza, rendendoli così idonei all'uso domestico o anche bellico.

Facendo un salto di diversi millenni, le nuove conoscenze che all'inizio del secolo scorso si venivano acquisendo sulla minerogenesi dei minerali che si trovano sulla superficie terrestre, ovvero che questi si fossero potuti formare solo nelle condizioni di pressione e temperature estreme che si trovano all'interno della terra, suscitarono un estremo interesse nel cercare di riprodurre in qualche modo quei processi anche in laboratorio. In particolare, si cercava di verificare se ad alte pressioni fosse possibile arrivare a sintetizzare nuovi materiali di interesse tecnologico, che fossero poi metastabili e perciò utilizzabili in condizioni normali. Un caso tipico riguarda la sintesi di materiali ultraduri, [11] a cominciare dal sogno più ambito, quello di riuscire a trasformare la forma allotropica più stabile del carbonio a c.n., la grafite, nella sua forma più preziosa, il diamante. Il sogno fu coronato, operando alla pressione di $10 \mathrm{GPa}$, alla temperatura di circa $2000 \mathrm{~K}$ ed in presenza di solfuro di ferro fuso come catalizzatore, da B. von Platen in Danimarca nel 1953 e, appena poco dopo, nel 1954, da H. Tracy Hall negli Stati Uniti, dopo più di un secolo di tentativi infruttuosi. [12] Realizzare questo sogno significò aver trovato il modo di convertire un solido conduttore, facilmente sfaldabile, con una struttura costituita da strati planari di atomi di $\mathrm{C}$ e tenuti insieme da deboli interazioni di van der Waals, in un materiale isolante, durissimo e con una struttura tridimensionale di legame. È interessante notare che la variazione strutturale da solido bidimensionale a tridimensionale, da facilmente sfaldabile a ultraduro, da conduttore a isolante, si accompagna ad una drastica variazione, indotta dalla pressione applicata, della natura del legame carbonio-carbonio, attraverso una modifica dello stato di ibridizzazione del carbonio, da planare $s p^{2}$ a tetraedrico $s p^{3}$. L'applicare una pressione esterna elevata porta dunque a significative modifiche della distribuzione elettronica degli atomi di un materiale. Si noti che il diamante è metastabile in c.n., perché stabile solo a livello cinetico e non termodi- 
namico. Ovvero, è separato da una barriera energetica così grande per la sua riconversione in grafite che, ottenuto o formatosi ad alte $\mathrm{P}$ e poi riportato a c.n., si mantiene inalterato nel tempo e quindi perfettamente utilizzabile per le sue innumerevoli applicazioni tecnologiche e ornamentali.

Importanti applicazioni dell'alta pressione si ebbero, a partire dal secolo scorso, anche in campo biologico. Nel 1915 il fisico Percy William Bridgman pubblica una breve nota per descrivere un fatto di potenziale interesse biologico, dichiarando umilmente di averlo scoperto in modo del tutto incidentale e nel corso di un lavoro con ben diverse finalità. [13] Bridgman aveva osservato che "se il bianco d'uovo è soggetto a una pressione idrostatica a temperatura ambiente, coagula, presentando un aspetto proprio come un uovo sodo". Alle pressioni utilizzate da Bridgman, circa 0.7 GPa, l'effetto della temperatura è limitato ed inaspettato, in quanto la facilità di coaugulazione aumenta abbassando la temperatura e viene invece ritardata, se non inibita, innalzandola. Di fatto Bridgman aveva scoperto che il passaggio da una proteina nativa ad una proteina denaturata può essere provocato sia da variazioni di temperatura, sfruttate usualmente nella cucina di casa per rassodare l'uovo, che da variazioni di pressione. Dalla sua scoperta, prendono l'avvio le diverse applicazioni delle alte pressioni nella scienza degli alimenti. [14-15] Ad esempio, i microorganismi nocivi possono essere distrutti ad alte pressioni invece che con un trattamento termico, preservando meglio le qualità organolettiche degli alimenti.

\subsection{Come raggiungere alte pressioni: la cella ad incudine di diamante}

Bridgman riceverà poi il premio Nobel per la fisica nel 1946, non tanto per la sua scoperta accidentale in ambito biologico, quanto per il suo contributo fondamentale alla fisica delle alte pressioni. In particolare Bridgman fu premiato per le macchine che aveva progettato, capaci di generare pressioni idrostatiche fino a $10 \mathrm{GPa}$ e successivamente anche a $30 \mathrm{GPa}$. Il suo è stato un modello primitivo di cella ad incudine, che ora, insieme alle onde d'urto, è lo strumento principe per lo studio della materia ad alte pressioni. [16-17] Il principio della cella ad incudine è quello del "tacco 12". Se non desideriamo sprofondare camminando in neve fresca, calziamo le ciaspole; se invece vogliamo sprofondare, niente di meglio che indossare un tacco 12, il quale esercita una pressione locale enorme, di 2-3 ordini di grandezza superiore a quella 
delle ciaspole (per convincersene, se non è possibile fare la prova pratica, basta considerare i rapporti delle aree di appoggio). Se contrapponiamo un tacco 12 ad un altro e se poi sostituiamo i tacchi con delle punte micrometriche di diamante, ricreiamo il principio della diamond anvil cell (DAC), [16-18] la cella di compressione ad incudine di diamante, in grado di esercitare pressioni dell'ordine delle centinaia di GPa. La DAC, schematizzata in Fig. 2, è costituita, oltre che dalle due punte che si contrappongono, da tre componenti principali: a) un dispositivo per generare la forza di compressione, per mezzo di viti di serraggio ("screws"), che esercitano una forza su ciascuna delle basi delle due punte; b) un mezzo di trasmissione della pressione (Ar, Xe, He, olio di paraffina, miscela di metanolo ed etanolo, etc.), che trasforma la pressione uniassiale esercitata dalle punte di diamante in una pressione idrostatica uniforme sul campione da analizzare; c) una guarnizione ("gasket") metallica, a guisa di cella, che racchiude il campione, il mezzo di trasmissione e gli estremi delle punte di diamante.

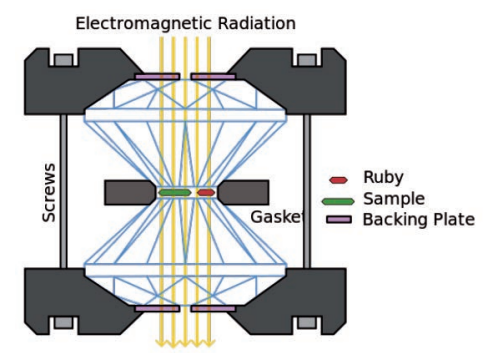

Fig. 2. Cella ad incudine di diamante (Tobias 1984, rif. [18]).

Il campione può essere poi analizzato, illuminandolo con raggi $\mathrm{X}$ o luce visibile, attraverso i diamanti che sono sostanzialmente trasparenti a queste radiazioni (nel caso della radiazione $\mathrm{X}$, la guarnizione metallica viene sostituita da una guarnizione di materiali più leggeri, anch'essi trasparenti ai raggi $\mathrm{X}$ ). Vengono utilizzate svariate tecniche, quali la diffrazione e fluorescenza di raggi X, l'assorbimento ottico e la fotoluminescenza, le spettroscopie di scattering Mössbauer, Raman e Brillouin...La pressione agente sul campione è misurata tramite un materiale di riferimento di cui sia noto un particolare comportamento al variare della pressione, ad es. lo spostamento a lunghezze d'onda più elevate delle due linee di fluorescenza del rubino ("ruby", Fig. 2) all'aumentare della pressione. 


\section{PRESSIONE, PERIODICITÀ E PROPRIETÀ}

La periodicità delle proprietà degli elementi che costituiscono un composto chimico ci guidano nella descrizione ed interpretazione della sua struttura geometrica ed elettronica ed anche della sua reattività. In modo più o meno conscio, il progressivo disvelarsi di questa periodicità ha fatto da guida alla proposizione della Tavola Periodica (TP), poi razionalizzata appieno grazie alle conoscenze via via acquisite sulla struttura elettronica atomica (si veda a tal proposito il testo della conferenza del Prof. Carlo Maria Prosperi). Tra le diverse proprietà periodiche, una delle maggiormente influenzate dall'effetto della pressione è il raggio atomico. Un aumento di pressione porta ad una riduzione del volume che una sostanza può occupare e di conseguenza ad una riduzione del volume degli atomi che la costituiscono. Come vedremo, però, la diminuzione percentuale di raggio atomico all'aumentare della pressione non è eguale per tutti gli elementi e ciò comporta variazioni evidenti delle usuali nozioni di periodicità. A pressione ambiente i raggi atomici aumentano in valore muovendosi dall'alto verso il basso lungo un gruppo della TP, mentre diminuiscono muovendosi da sinistra verso destra lungo un periodo della TP, all'interno dei blocchi $s$ e $p$ (Fig. 3). Questi andamenti non sono che la conseguenza della struttura elettronica degli atomi, che si riflette nella loro specifica posizione nella TP (Fig. 3). Scendendo lungo un gruppo, gli elettroni esterni sono in orbitali di numero quantico principale $n$ crescente e pertanto hanno una distanza media maggiore dal nucleo. Lungo un periodo gli elettroni di valenza occupano orbitali dello stesso guscio principale, ma la carica nucleare efficace cresce lungo il periodo, per cui gli elettroni esterni sentono una carica nucleare progressivamente sempre meno schermata rispetto a quella percepita dal singolo elettrone esterno dei metalli alcalini, risultando in atomi progressivamente sempre più compatti. La periodicità dei raggi atomici a pressioni ordinaria, $10^{-4} \mathrm{GPa}$, riflette la periodicità delle configurazioni elettroniche del guscio atomico più esterno. La diversità dei raggi differenzia le proprietà chimiche e di conseguenza le strutture e proprietà dei solidi elementari e dei loro composti. Ad esempio, i metalli alcalini sono costituiti da elementi con un solo elettrone legato molto debolmente nel loro guscio $s$ esterno e hanno pertanto raggi atomici molto grandi, mentre gli elementi dei metalli di transizione sono caratterizzati da elettroni esterni per lo più in orbitali $d$, molto meno schermati, e conseguentemente raggi atomici molto più piccoli. 


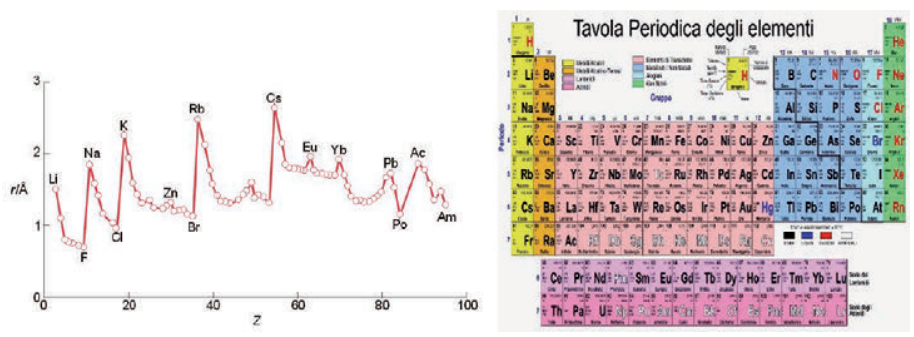

Fig. 3. La periodicità dei raggi degli atomi in funzione del numero atomico $\mathrm{Z}$ riflette la configurazione elettronica dei loro gusci più esterni. Quest'ultima determina il loro posizionamento nella Tavola Periodica.

\subsection{Il drammatico effetto della pressione sui raggi atomici}

Sotto l'azione di una pressione esterna, lo scenario delineato in Fig. 3 cambia drasticamente. Quando un atomo viene compresso, il valor medio della sua densità elettronica (elettroni per unità di volume) aumenta ed il guscio esterno risulta il più facilmente comprimibile. Già a 100 GPa (Fig. 4) la variazione di raggio atomico lungo un periodo della TP diventa molto meno evidente ed è, ad esempio, difficile distinguere i metalli alcalini dai metalli di transizione. A $1000 \mathrm{GPa}$ la nozione di periodicità dei raggi atomici si perde definitivamente. Ciò implica che la natura dei legami chimici ne sia significativamente influenzata e possa dar luogo a composti e strutture cristalline inusuali e con proprietà spesso inaspettate.

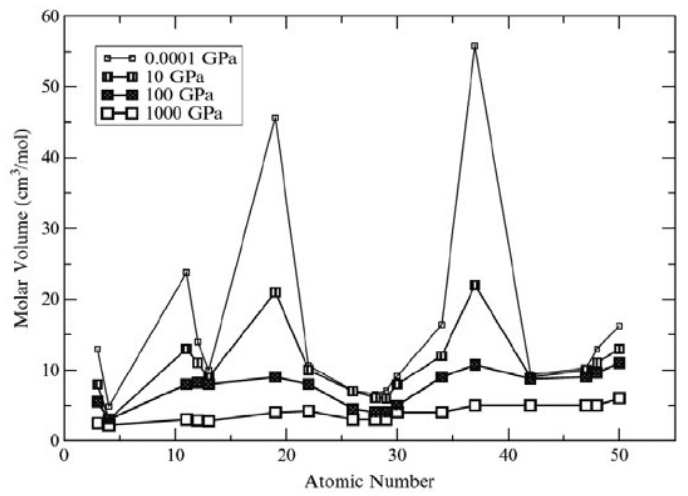

Fig. 4. Volumi molari dei solidi elementari in funzione del numero atomico Z. I raggi atomici vengono derivati utilizzando per gli atomi nel cristallo un modello a impaccamento compatto di sfere (riprodotta con permesso da Fig. 17.1 del Rif. [19]). 


\subsection{L'energia dei legami chimici vs il lavoro di pressione su un sistema}

È anche opportuno esaminare gli effetti della pressione su un sistema da una prospettiva energetica, confrontando il lavoro fatto per comprimerlo, PV, rispetto alle tipiche forze di legame. [19] Il lavoro termodinamico di compressione sul sistema equivale all'energia che il sistema, modificandosi, acquisisce all'equilibrio per reagire alla pressione esterna (nell'espressione PV consideriamo anche il volume $\mathrm{V}$ come grandezza intensiva, utilizzando ad esempio il volume molare). A basse pressioni, sotto i $10 \mathrm{GPa}$, il lavoro di compressione, ovvero l'energia acquisita dal sistema, è confrontabile con l'energia di un legame a idrogeno di forza media (circa $2-10 \mathrm{Kcal} / \mathrm{mole}$ ). In Fig. 5 a vediamo tratteggiato il legame ad idrogeno nel dimero dell'acqua (circa $5 \mathrm{Kcal} / \mathrm{mole}$ ). A pressioni più elevate, intorno ai $100 \mathrm{GPa}$, il lavoro di compressione diventa confrontabile con l'energia di un usuale legame chimico covalente (circa $100 \mathrm{Kcal} / \mathrm{mole}$ ). Sulla forma esagonale del ghiaccio, che è la fase $\left(I_{b}\right)$ stabile a pressione ambiente, una pressione dell'ordine dei 50$100 \mathrm{GPa}$ avrà effetto non solo sui legami a idrogeno, ma anche sui legami covalenti polari $\mathrm{O}-\mathrm{H}$ (Fig. 5b). In effetti già a $60 \mathrm{GPa}$ il ghiaccio diventa stabile nella sua fase $X$ (Fig. 5 c), in cui i doppietti isolati (lone pairs) degli atomi di ossigeno non sono più coinvolti in legami ad idrogeno, ma condivisi a formare nuovi legami O-H. Di fatto nella fase $X$ non è più possibile distinguere legami intramolecolari e intermolecolari, in quanto tutti i legami O-H diventano equivalenti, di natura covalente-polare, e non è più possibile individuare le molecole d'acqua nella struttura del cristallo. Ogni ponte O-H...O del ghiaccio $I_{b}$ è divenuto nella fase $X$ un ponte simmetrico $\mathrm{O}-\mathrm{H}-\mathrm{O}$, tenuto insieme da un legame a tre centri ricco in elettroni.

L'effetto primario di un aumento della pressione è la riduzione del volume, la quale può essere conseguita anche diminuendo la temperatura. La riduzione percentuale del volume ottenibile con un aumento della pressione è però decisamente più significativa. [6] Una pressione di $200 \mathrm{GPa}$ porta ad una riduzione di un fattore 1.5-5 del volume di un solido, pari, nel caso di una compressione isotropica, ad un raccorciamento delle distanze interatomiche di un fattore 1.1-1.7. Sono effetti chiaramente ben visibili sulle proprietà di un solido. Ad esempio, il cristallo atomico dello Xeno, che fonde a $112^{\circ} \mathrm{C}$ alla pressione di un'atmosfera, diviene a $50 \mathrm{GPa}$ un solido estremamente compatto, con un punto di fusione di $3000^{\circ} \mathrm{C}$. Sono variazioni che portano anche a dra- 
stiche evoluzioni strutturali, come abbiamo già visto nel caso della trasformazione da ghiaccio $I_{b}$ a ghiaccio $X$. La necessità di queste variazioni può spesso essere facilmente compresa. Se per esempio si comprime il cristallo di $\mathrm{SiH}_{4}$ mantenendo artificialmente la sua struttura tetraedrica attraverso un esperimento puramente computazionale, ben presto si arriva a contatti $\mathrm{H} \mathrm{H}$ che, essendo troppo ravvicinati, divengono progressivamente insostenibili da un punto di vista energetico, malgrado l'energia fornita al sistema dal lavoro di compressione. È pertanto una situazione che, non appena venisse rimosso il vincolo della simmetria tetraedrica, porterebbe inevitabilmente ad una evoluzione strutturale significativa. [6]
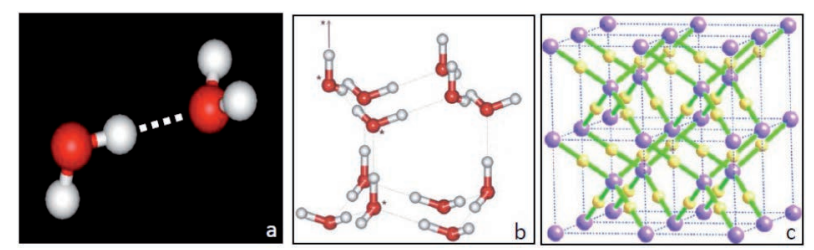

Fig. 5. Effetto della pressione sul legame ad idrogeno dell'acqua: a) nel dimero dell'acqua (O sfera rossa, $\mathrm{H}$ sfera bianca) il legame ad idrogeno, tratteggiato in bianco, è chiaramente distinguibile dal legame intramolecolare $\mathrm{O}-\mathrm{H}$; b) fase esagonale del ghiaccio, $I_{b}$, stabile a pressione ambiente; c) a $60 \mathrm{GPa}$ la fase esagonale si trasforma nella fase $X$, in cui non è più possibile distinguere tra legami $\mathrm{O}-\mathrm{H}$ e legami a idrogeno $(\mathrm{H}$ giallo, $\mathrm{O}$ violetto) (Fig $5 a$ e $5 b$ riprodotte da https://en.wikipedia.org/wiki/File:Water_dimer.jpg e da http://www1.lsbu.ac.uk/water/hexagonal_ice.html; Fig. 5c da Fig. 3 di Rif. [6]).

\subsection{Termodinamica chimica ed evoluzione di un sistema sotto pressione}

All'aumentare della pressione, un sistema può accedere a configurazioni che non gli sono normalmente accessibili a pressione ordinaria. Per capire più a fondo questo aspetto, dobbiamo far ricorso a nozioni elementari di termodinamica chimica. Un sistema all'equilibrio è caratterizzato da un valore minimo di energia libera $\mathrm{G}=\mathrm{H}+\mathrm{TS}$, dove $\mathrm{H}$ è l'entalpia del sistema, data dalla somma $\mathrm{H}=\mathrm{U}+\mathrm{PV}$, U è la sua energia interna e PV è il termine legato alle forze esterne. S è l'entropia e T la temperatura. L'energia interna di un sistema è definita come la sua energia totale, ad esclusione dell'energia cinetica del sistema considerato nel suo insieme (moto di traslazione del centro di massa) e dell'energia potenziale derivante dall'interazione con forze esterne. Per semplicità ci 
limitiamo nel nostro ragionamento a $\mathrm{T}=0$, per cui $\mathrm{G}$ coinciderà con $\mathrm{H}$. In queste condizioni, il sistema evolverà verso l'equilibrio termodinamico minimizzando la sua entalpia. Pertanto, all'aumentare della pressione, la situazione di equilibrio del sistema non sarà più quella usuale a $\mathrm{P}$ ambiente, in quanto comincia a pesare il termine PV che è sostanzialmente zero a $\mathrm{P}$ atmosferica. Il sistema dovrà quindi minimizzare la sua entalpia, cercando un compromesso tra aumento di energia interna, dovuto a configurazioni intrinsecamente meno favorevoli rispetto a quella adottata a pressione ordinaria, e minimizzazione del termine PV. Dato un certo valore della pressione, il sistema tenderà ad acquisire una configurazione geometrica, ovvero una disposizione dei suoi nuclei ed una distribuzione elettronica, tale da minimizzarne il volume. Il termine PV acquisisce progressivamente importanza all'aumentare della pressione, fino a poter divenire dominante nel bilancio energetico. Il sistema, cercando di opporsi alla compressione esterna, evolverà verso strutture sempre più compatte ma non sempre corrispondenti a quelle previste dal close-packing, ovvero dall'impaccamento compatto di sfere.

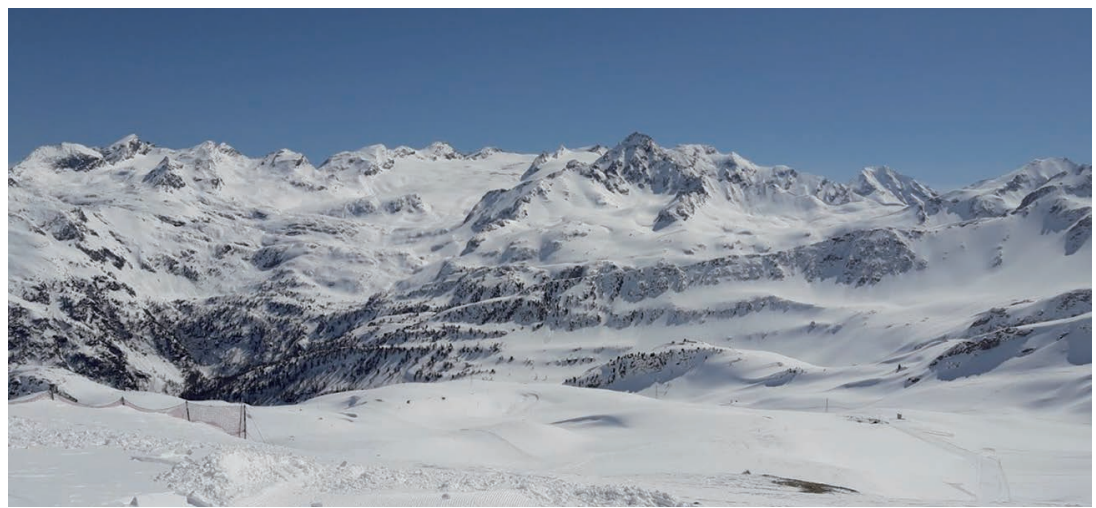

Fig. 6. L'aumento della pressione permette ad un sistema di esplorare regioni di energia potenziale ad esso altrimenti inaccessibili, muovendosi progressivamente dai minimi di fondovalle verso le alte valli alpine e le cime più elevate (fotografia dell'autore).

La minimizzazione dell'entalpia e il tentativo di opporsi alla pressione esterna porta un sistema ad esplorare mondi per esso nuovi. Possiamo visualizzarlo utilizzando l'immagine di un panorama alpino (Fig. 6). Se ad ogni punto della superficie rappresentata nella figura associamo una configurazione nucleare $X$ del sistema ed un valore di $\mathrm{U}(X)$ pari all'altitudine del punto, all'aumentare della $\mathrm{P}$ il sistema si muoverà 
dal fondovalle, ovvero dai minimi di U, a punti a maggiore altitudine, caratterizzati da nuove configurazioni in cui, pur aumentando la sua energia interna, il sistema riesce a minimizzare la sua entalpia, adottando configurazioni sempre più compresse $[$ ad una data $\mathrm{P} \rightarrow \min (\mathrm{U}(X)+\mathrm{PV}(X))]$. Il sistema si muoverà progressivamente verso le cime della montagna, andando a visitare regioni di spazio configurazionale prima inaccessibili e caratterizzate da strutture e legami chimici del tutto inusuali.

\section{CHIMICA SOTTO PRESSIONE: QUALCHE ESEMPIO}

Dopo la carrellata di informazioni generali presentate nei capitoli precedenti e prima di qualche considerazione conclusiva, desidero presentare brevemente alcuni esempi di evoluzione di cristalli sotto pressione. Inizierò da alcuni semplici cristalli molecolari, illustrerò alcune regole generali, evidenziandone i limiti, per poi presentare tre casi di lavori molto recenti, che riguardano sistemi che a pressioni ordinaria si possono classificare rispettivamente come un solido molecolare, il boro, come un tipico metallo, il sodio, ed infine come il prototipo dei cristalli ionici, il sale da cucina $(\mathrm{NaCl})$. Queste nette classificazioni, come vedremo, vengono completamente a cadere all'aumentare della pressione: il boro diventa parzialmente ionico, [20-21] il sodio diviene un isolante [22] ed il sale da cucina si trasforma, a seconda del valore di pressione, in strutture con proprietà diverse e rapporti stechiometrici tra gli atomi del tutto impensabili. [23-24] Da ultimo presenterò il sistema dell'eliuro di sodio $\mathrm{Na}_{2} \mathrm{He}$, primo composto dell'elio termodinamicamente stabile, seppur ad alte pressioni. [25] A due di queste nuove scoperte, ovvero la nuova fase ionica del $\mathrm{B}$ e la proposizione di $\mathrm{Na}_{2} \mathrm{He}$, ho avuto la possibilità di contribuire personalmente.

\subsection{Cristalli molecolari}

Il comportamento dei cristalli molecolari all'aumentare della pressione presenta alcuni tratti comuni: a) gli atomi che li costituiscono aumentano la loro coordinazione media; b) nel caso vi siano legami multipli, questi tendono a rompersi a vantaggio di una maggior coordinazione atomica, purché non si debbano superare barriere energetiche eccessive; c) si formano fasi estese, per adattarsi alla maggiore densità gravimetrica, costituite da polimeri mono-, bi- e anche tri-dimensionali, 
con proprietà spesso molto diverse da quelle dei solidi molecolari di partenza. [6]

Il caso dello $\mathrm{S}$ elementare è a questo proposito paradigmatico (Fig. 7). A pressione ambiente (fase I) è un solido molecolare, un nonmetallo costituito da molecole ad anello $\mathrm{S}_{8}$ con gli atomi di zolfo disposti a corona, distanze S-S pari a $2.04 \AA$ (1 Angstrom, simbolo $\AA$, è pari a $10^{-8} \mathrm{~cm}$ ) e distanze di non legame $\mathrm{S} . . \mathrm{S}$ comprese tra 3.37 e $3.50 \AA$. La fase I si trasforma a pressioni $>1.5 \mathrm{GPa}$ nella fase II, un polimero infinito monodimensionale che consiste di catene elicoidali con passo 3 , un'invariata distanza $S-S$ e una distanza più corta tra atomi non legati (S...S, $3.2 \AA$ ). Quando la pressione supera i $36 \mathrm{GPa}$, la fase II si trasforma nella fase III, costituita da eliche infinite con un asse elicogiro quaternario, legami $S$-S più lunghi $(2.09 \AA)$ e contatti secondari $\mathrm{S}$...S più ravvicinati (3.02 $\AA$ ). La fase IV, che diviene stabile a pressioni superiori a $83 \mathrm{GPa}$, è formata da strati corrugati di atomi di zolfo, con coordinazioni $(2+2+4)$ o $(2+4+4)$, ben lontane dalla coordinazione 2 delle fasi precedenti e con i lone pairs dello zolfo significativamente impiegati nel legame chimico S-S. Questo progressivo sconvolgimento strutturale e di legame fa sì che lo $\mathrm{S}$ elementare diventi nella sua fase IV un metallo con proprietà di superconduttore. [26]

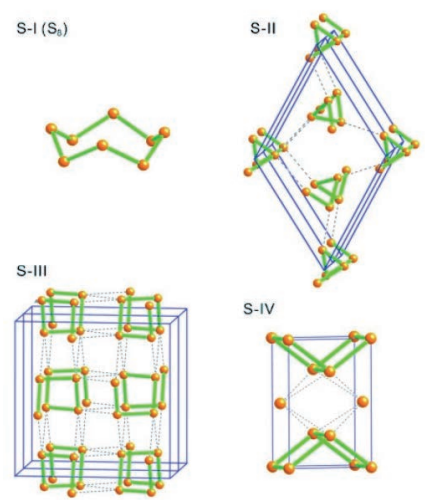

Fig 7. Strutture cristalline dello zolfo (S) a varie pressioni e temperature (fasi I, II III e IV). Le interazioni di legame secondarie sono indicate con una linea tratteggiata. Riprodotta da Fig. 4 del Rif. [6].

Nelle molecole $\mathrm{CO}_{2}$ e $\mathrm{N}_{2}$ gli atomi sono legati attraverso il legame doppio $\mathrm{C}=\mathrm{O}$ ed il legame triplo $\mathrm{N}^{\circ} \mathrm{N}$. Questi sono in natura i legami chimici più forti, con energie di legame pari rispettivamente a $187 \mathrm{Kcal} / \mathrm{mol}$ 
e $226 \mathrm{Kcal} /$ mole. Malgrado siano dei veri pozzi termodinamici, questi legami sono insaturi e la loro insaturazione viene presto violata ad alta pressione. [6] La molecola lineare della $\mathrm{CO}_{2}$ a $\mathrm{P}>35 \mathrm{GPa}$ polimerizza formando un solido esteso (fase $\mathrm{V}$ ) con il $\mathrm{C}$ in arrangiamento quasi tetraedrico e con una struttura analoga a quella di uno dei polimorfi della silice a pressioni ordinarie (Fig. 8). Analogamente, nella struttura cubica-gauche dell'azoto (cg-N), l'atomo di azoto diventa tri-coordinato, come nella molecola di ammoniaca (Fig. 8). Si forma una fase estesa con legami singoli covalenti N-N, che è molto simile a quella del fosforo solido. La tendenza da parte di un elemento a formare ad alta pressione strutture analoghe a quelle dell'elemento successivo del suo gruppo a pressione ordinaria è una situazione che in effetti si riscontra molto frequentemente.
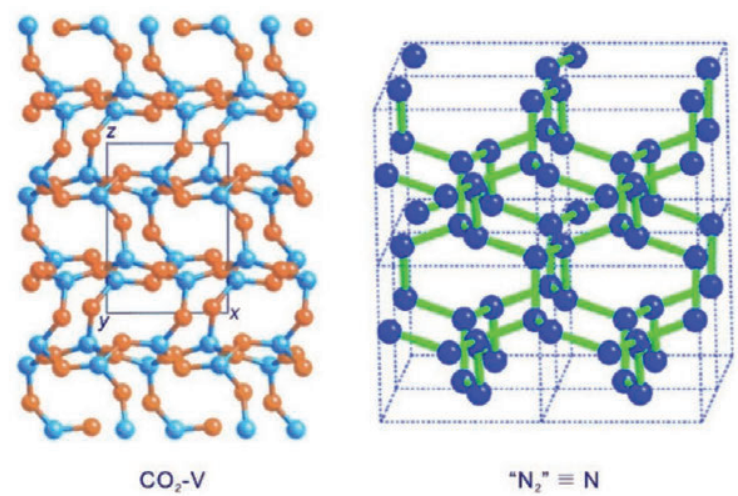

Fig. 8. Strutture cristalline della $\mathrm{CO}_{2}$ polimerica (fase $\mathrm{V}, \mathrm{C}$ blu, $\mathrm{O}$ arancio) e di $\mathrm{N}_{2}$ polimerico (fase cg-N). Riprodotta da Fig. 4 del Rif. [6].

La struttura cg- $\mathrm{N}$ è metastabile a pressioni ordinarie ed è una forma di azoto polimerico tridimensionale, con una durezza paragonabile a quella del diamante. Poiché il legame singolo N-N ha un'energia molto bassa (circa $38 \mathrm{Kcal} /$ mole), l'energia che si può liberare trasformando un azoto tricoordinato in un azoto monocoordinato con legame triplo è pari a $56 \mathrm{kcal}$ per atomo di azoto $(0.5 * 226 \mathrm{Kcal} / \mathrm{mole}-$ $0.5 * 38 * 3 \mathrm{Kcal} / \mathrm{mole})$. L'azoto polimerico, archetipo dei materiali energetici, ha quindi una capacità energetica enorme, cinque volte maggiore dei migliori materiali energetici sinora proposti. [5] Le sue eccezionali proprietà erano già state predette teoricamente da Mailhiot et al. [27] negli anni Ottanta, ma solo all'inizio di questo secolo si è potuto con- 
fermarle sperimentalmente, operando a $120 \mathrm{GPa}$ e a temperature elevatissime, 1900 K. [28] L'azoto polimerico rappresenta una scoperta estremamente promettente, soprattutto quando si riuscisse ad ottenerlo in condizioni più agevoli ed economicamente meno impegnative e quando venissero pienamente risolte le questioni legate al suo impiego, in condizioni controllate, a pressione ordinaria. Il suo utilizzo sarebbe del tutto ecologico, in quanto verrebbe convertito in azoto molecolare, che costituisce il $78 \%$ in volume dei gas atmosferici. [5]

\subsection{Regole generali e loro limiti}

Malgrado vadano accumulandosi studi sperimentali e computazionali sui sistemi sotto pressione, non si è ancora giunti ad una razionalizzazione completa e soddisfacente di quello che può effettivamente avvenire in queste situazioni. Ovvero, senza effettuare un esperimento pratico o un'indagine computazionale approfondita, è attualmente molto difficile, se non impossibile, fare delle previsioni dettagliate sull'evoluzione di un sistema progressivamente compresso. Vi è ancora troppo poca esperienza, in rapporto ai gradi di libertà disponibili e alle loro diverse valenze energetiche. A seconda del tipo di atomo, della sua configurazione elettronica esterna ( $\mathrm{ma}$ ad alte $\mathrm{P}$ anche di quella immediatamente più interna), della presenza e dell'interazione con atomi diversi, del grado di pressione esterna, divengono possibili moltissime evoluzioni strutturali ed elettroniche, con energie a volte comparabili e con scale di stabilità relativa che possono variare sensibilmente all'aumentare della pressione. In molti casi, quelle che sembravano regole assodate sono state successivamente smentite. Roald Hoffmann et al. [6] ne hanno elencate alcune, riportandole in ordine di energia e, conseguentemente, di pressione crescente. Questo comporta che in uno stesso sistema possono essere seguite diverse regole contemporaneamente e con pesi energetici variabili a seconda dell'intervallo di pressione.

Eccone un breve riassunto: [6]

a) vengono inizialmente penetrate le regioni repulsive associate ai potenziali intermolecolari, ovvero lo spazio van der Waals è quello che viene più facilmente compresso;

b) le strutture ioniche e covalenti, siano esse solidi molecolari o solidi estesi, reagiscono alla pressione aumentando la coordinazione atomica e adottando generalmente il legame multicentrico quale modalità di legame per rendere le strutture più compatte; 
c) la lunghezza dei legami covalenti diminuisce; gli anioni vengono compressi molto più facilmente dei cationi, così che la coordinazione atomica, specie per i cationi, aumenta con la pressione;

d) tutti i semiconduttori e gli isolanti diventano metallici a pressione sufficientemente elevata, ma, come vedremo per il sodio, può verificarsi anche la situazione opposta, ovvero metalli che si trasformano in isolanti;

e) la regola che viene normalmente seguita è il close packing, l'impacchettamento ottimale di "sfere" atomiche, ma, ad ulteriori innalzamenti di pressione, il sistema persegue un impacchettamento ancora più denso, grazie ad un disproporzionamento elettronico e a una sostanziale deformazione delle densità elettroniche atomiche pseudo sferiche. Vedremo questo comportamento negli esempi del boro e del salgemma ad alta pressione;

f) gli elettroni più esterni possono lasciare i loro atomi originari, localizzandosi in siti interstiziali, e formando essi stessi dei reticoli regolari con schemi di legame non più nucleo-centrici, ma elettrone-centrici e basati su elettroni interstiziali altamente localizzati. Ne vedremo degli esempi nel caso del sodio e dell'eliuro di sodio, $\mathrm{Na}_{2} \mathrm{He}$;

g) la pressione può progressivamente mettere in gioco orbitali più interni, che un chimico non penserebbe mai di coinvolgere in legami chimici.

\subsection{Il caso della doppia personalità del Boro sotto pressione}

Il Boro è sicuramente un elemento particolare ed affascinante. [2930] Ha proprietà eccezionali, una bassa volatilità, un alto punto di fusione $\left(2450^{\circ} \mathrm{C}\right)$, è più forte dell'acciaio, più duro del corindone (corindone: durezza 9) e più leggero dell'alluminio. A pressione ambiente è il primo elemento solido non metallico ed è l'unico non metallo del suo gruppo. Analogamente al carbonio, l'elemento che lo segue nella TP, ed al silicio, elemento contiguo diagonale nella TP, ha tendenza a formare legami covalenti, ma differisce fortemente da questi due elementi perché ha solo tre elettroni di valenza, ovvero un elettrone in meno dei suoi orbitali di valenza. Questa peculiarità, comunemente classificata come deficienza elettronica, lo porta ad essere estremamente sensibile ad essere drogato con impurezze e rende il suo studio non solo difficile, ma anche pieno di insidie. Di fatto quale sia la sua fase stabile non è stato ancora ben chiarito nemmeno in condizioni ambientali standard [31] e solo recentemente la 
si è forse potuta definire a livello teorico. Il Boro può essere considerato come un autentico cimitero per la reputazione di molti chimici, anche illustri. [29] Si inizia nel 1808, quando J. L. Gay-Lussac e L. J. Thénard a Parigi e Sir Humphrey Davy a Londra riportano, indipendentemente, di aver scoperto un nuovo elemento, il Boro. Pochi anni dopo si ipotizza che in entrambi i casi si tratti in realtà di un suo composto, con percentuali di Boro non superiori al 60-70\%. Si deve aspettare comunque il 1895 ed un altro grande chimico, H. Moissan, per una prova definitiva di questa ipotesi e per una nuova "scoperta" dell'elemento Boro. Ma dopo pochi anni anche l'elemento scoperto da Moissan si rivela un composto contenente solo il 90\% di Boro. Nel 1858, nel suo libro di testo Elements de Chimie Inorganique Et Organique, [32] F. Wöhler riporta che il Boro ha due polimorfi, uno tipo grafite ed uno simile al diamante. Ora però sappiamo che queste due forme sono in effetti due composti chimici, rispettivamente $\mathrm{AlB}_{12}$ e $\mathrm{B}_{48} \mathrm{C}_{2} \mathrm{Al}$. Nel 1909 , viene finalmente sintetizzato il B con una purezza del 99\%. [29] Ma anche questa purezza non rappresenta la fine del mistero del Boro, in quanto l'1\% di impurezza, o anche meno, è sufficiente per cambiarne in modo estremamente significativo struttura e proprietà. Indipendentemente dalla loro purezza, tutte le forme note del Boro contengono come mattone strutturale il cluster icosaedrico $\mathrm{B}_{12}$ (Fig. 9) con legami policentrici, quasi tipo metallo, all'interno degli icosaedri e legami bicentrici o tricentrici tra icosaedri. Questa struttura di legame soddisfa la regola dell'ottetto e dà luogo ad uno stato isolante, ma le fasi drogate con impurezze sono innumerevoli ed in genere metalliche $\left(\mathrm{YB}_{65}, \mathrm{NaB}_{15}, \mathrm{MgAlB}_{14}, \mathrm{AlC}_{4} \mathrm{~B}_{40}, \mathrm{NiB}_{50}, \mathrm{PuB}_{100}\right)$. Di fatto solo tre delle innumerevoli fasi descritte in letteratura per il Boro corrispondono all'elemento puro: le fasi romboedriche $\alpha-B_{12}$ e $\beta-B_{106}$ con 12 e 106 atomi per cella elementare e la fase tetragonale T-192, con 190-192 atomi per cella elementare. A pressioni ordinarie la fase tetragonale è la più stabile ad alte temperature, mentre a temperatura ambiente le due fasi romboedriche hanno stabilità statiche simili e solo la considerazione dei moti vibrazionali di punto zero sembra indicare la fase $\beta-\mathrm{B}_{106}$ come la più stabile. Innalzando la pressione, già a pochi GPa la fase $\alpha-B_{12}$, più densa, diventa la fase del Boro più stabile. Ma cosa succede a pressioni esterne maggiori? Si può ipotizzare che due effetti possano contrapporsi. L'alta pressione dovrebbe favorire uno stato metallico, stabilizzando i cluster icosaedrici che già sono caratterizzati da un legame intra-icosaedrico di tipo quasi metallico a pressioni ordinarie; d'altra parte l'impaccamento atomico delle strutture icosaedriche è estremamente poco efficiente, 
tanto che, ad esempio, solo il $34 \%$ del volume viene utilizzato in $\alpha-\mathrm{B}_{12}$. Si può pertanto immaginare che ad un certo punto il sistema debba necessariamente trasformarsi, distruggendo i suoi mattoni icosaedrici e formando fasi più dense, in grado di contrastare, tramite la diminuzione di volume, l'aumento progressivo della pressione esterna. Il mistero dell'evoluzione strutturale del Boro all'aumentare della pressione è stato risolto in uno studio pubblicato su Nature nel 2009, a cui ho avuto il piacere e l'onore di contribuire e nel quale esperimento e teoria si sono sinergicamente incontrati. [20] La trattazione tecnica [20,33] del come si è arrivati a scoprirne il mistero esula dallo scopo del presente scritto, per cui limiterò l'attenzione ad alcuni aspetti legati agli effetti strabilianti della pressione. Partendo da campioni di $\beta-B_{106}$ di purezza 99,9999\% e innalzando progressivamente la pressione, la fase $\beta-\mathrm{B}_{106}$ presto si trasforma nella fase $\alpha-B_{12}$, che rimane stabile fino a circa $19 \mathrm{GPa}$, allorché diviene stabile una fase con caratteristiche inaspettate e prima del tutto sconosciuta (Fig. 9). La nuova fase, battezzata $\gamma$-boro, è ortorombica, ha 28 atomi per cella e può essere visualizzata a partire da una struttura tipo $\mathrm{NaCl}$, il sale da cucina, ma leggermente distorta. Sostituendo i cationi $\mathrm{Na}^{+}$ con dimeri $\mathrm{B}_{2}$ e gli anioni $\mathrm{Cl}^{-}$con icosaedri $\mathrm{B}_{12}$, si ottiene la struttura del $\gamma$-boro, $\gamma-B_{28}$. E' la forma più stabile del Boro tra 19 e $89 \mathrm{GPa}$, con i centri degli icosaedri $\mathrm{B}_{12}$ che danno origine ad un impaccamento cubico compatto leggermente distorto come nella fase $\alpha-B_{12}$. In $\gamma-B_{28}$, però, tutti i buchi ottaedrici della struttura cubica compatta vengono occupati dai dimeri $B_{2}$, rendendo questa nuova fase molto più densa e stabile di $\alpha-B_{12}$.

Sarebbe logico immaginare che l'analogia con $\mathrm{NaCl}$ si fermi all'aspetto strutturale, ma non è così. Come nel solido ionico $\mathrm{NaCl}$ vi è trasferimento di carica tra $\mathrm{Na} \mathrm{e} \mathrm{Cl}$, pari ad un elettrone, così si osserva un parziale trasferimento di carica tra dimeri $B_{2}$ e icosaedri $B_{12}$. Di fatto $\gamma-B_{28}$ può essere descritto come un boruro di boro $\left(\mathrm{B}_{2}\right)^{\delta+}\left(\mathrm{B}_{12}\right)^{\delta}$, con un trasferimento di carica $\delta$ tra cationi $B_{2}$ e anioni $B_{12}$ pari a circa 0.4 elettroni, che fa sì che $\gamma-B_{28}$ risulti parzialmente ionico e isolante fino a più di $200 \mathrm{GPa}$. L'aspetto più sconvolgente da un punto di vista chimico è l'aver scoperto una fase di un elemento in cui gli atomi costituenti non sono eguali tra loro, ma solo, ed approssimativamente per $\mathrm{B}_{12}$, all'interno dei clusters $\mathrm{B}_{2}$ e $\mathrm{B}_{12}$ tra cui avviene il trasferimento di carica. A prima vista ciò appare in aperta contraddizione con la nozione di elemento chimico che prevede uguali proprietà, inclusa un'uguale popolazione elettronica e una carica netta pari a zero, per ogni atomo di una sua fase. Questo è ad esempio ciò che si osserva per le fasi allotropiche del Carbonio: gli atomi di $\mathrm{C}$ nel diamante hanno proprietà 
diverse da quelli nella grafite, ma all'interno delle due fasi sono tra loro indistinguibili. Ma come abbiamo già accennato nella sezione 6.2, se l'evoluzione strutturale all'innalzarsi della pressione prevede in prima istanza di seguire la regola del close packing, ovvero dell'impacchettamento ottimale delle "sfere" atomiche, di fronte ad ulteriori aumenti di pressione il sistema può realizzare un impacchettamento ancora più denso grazie ad un disproporzionamento elettronico e ad una sostanziale deformazione delle densità elettroniche atomiche. Non deve pertanto sorprenderci il fatto che questo possa avvenire anche per le fasi di un elemento e sicuramente il caso del $\gamma$ Boro è uno di quelli, ad oggi scoperti, in cui ciò avviene in modo più eclatante. Il carattere parzialmente ionico di una fase elementare è stato oggetto di moltissimi commenti e riprese su varie testate molto autorevoli. Ho trovato però particolarmente arguto quanto riportato in un blog d'autore, ad opera di Teresa Celestino, [34] su un sito dedicato agli insegnanti di scuola superiore. L'autrice parla dello strano caso del Boro in termini del tema del doppio, così caro alla letteratura (i Menecmi di Plauto, le Metamorfosi di Ovidio, il Dottor Jekyll e Mister Hyde...), attribuendo al Boro una ben celata doppia personalità, elettrone attrattore ed elettrone donatore, in grado di sdoppiarsi ad alte pressioni, per poi auto-combinarsi in un sale elementare, il boruro di Boro.
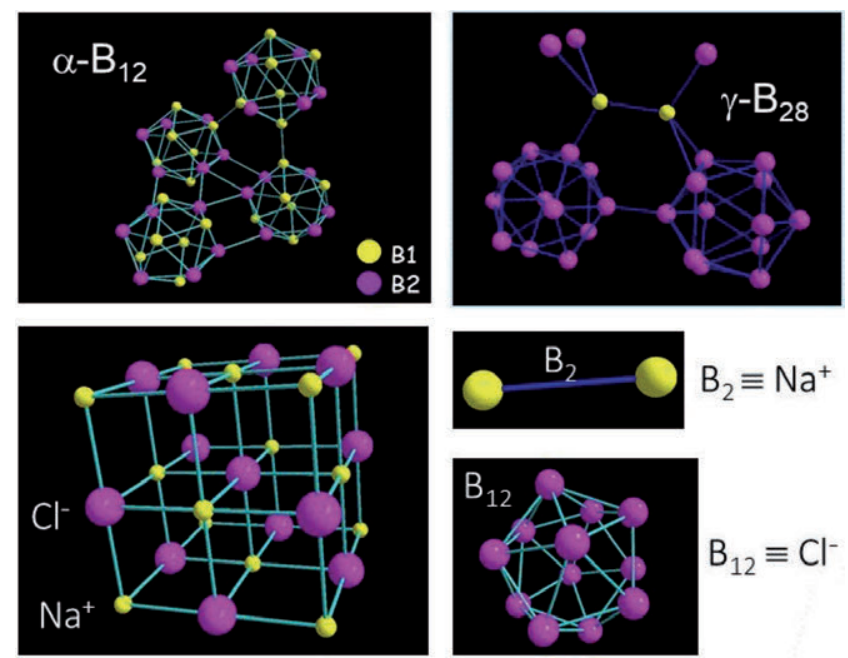

$$
\mathrm{B}_{12} \equiv \mathrm{Cl}^{-}
$$

Fig. 9. Elementi strutturali delle due fasi del Boro, $\alpha-B_{12}$ e $\gamma-B_{28}$. La struttura di $\gamma-B_{28}$ può essere visualizzata a partire da una struttura tipo $\mathrm{NaCl}$ (in basso a sinistra) ma leggermente distorta, in cui i dimeri $\mathrm{B}_{2}$ sostituiscono i cationi $\mathrm{Na}^{+}$e gli icosaedri $\mathrm{B}_{12}$ gli anioni $\mathrm{Cl}$. 


\subsection{Da conduttore ad isolante: il caso del Sodio}

All'innalzarsi della pressione, i metalli adottano distanze interatomiche sempre più corte, che comportano un aumento dell'ampiezza delle bande di conduzione e di valenza e, di conseguenza, un sempre più accentuato comportamento degli elettroni di valenza da elettroni quasi liberi. D'altra parte, alle densità gravimetriche che si possono raggiungere ai giorni nostri, la compressione può essere così grande da permettere una sovrapposizione degli orbitali degli elettroni più interni. In queste circostanze, si possono verificare comportamenti del tutto inusuali per un metallo. Il Sodio ha una struttura cubica a corpo centrato in condizioni ambientali standard. Sotto pressione si trasforma a $65 \mathrm{GPa}$ in una struttura cubica a facce centrate e a pressioni via via crescenti in una innumerevole serie di strutture sempre più compresse, ma comunque conduttrici, come il Sodio a pressione ordinaria. A circa $200 \mathrm{GPa}$ e ad un volume pari a 1/5 di quello a pressione ambiente, si osserva invece un fatto sorprendente. [22] Si forma una nuova fase, $\mathrm{Na}-\mathrm{hP} 4$, caratterizzata da una struttura d.h.c.p. (double-hexagonal close packed) altamente distorta (Fig. 10), con coordinazione sei attorno ad ogni atomo di $\mathrm{Na}$ e con un gap di banda estremamente elevato. Di conseguenza, diviene stabile una fase isolante e otticamente trasparente, invece che conduttrice, argentea e altamente riflettente come è la fase del Sodio a pressioni ordinarie. L'insorgenza della fase isolante non è dovuta alla formazione di coppie atomiche $\mathrm{Na}_{2}$ ma ad una ibridizzazione $p$ - $d$ degli elettroni di valenza del $\mathrm{Na}$ e ad una loro localizzazione negli spazi interstiziali del reticolo per minimizzare la repulsione con gli elettroni di cuore. Pertanto, la fase $\mathrm{Na}-\mathrm{hP} 4$ è formata da cuori ionici e coppie di elettroni localizzati e interstiziali, in perfetta analogia con il caso degli elettruri, e in cui i massimi di densità elettronica associati agli elettroni interstiziali di valenza giocano il ruolo degli anioni (Fig. 10). Non è difficile ipotizzare che stati isolanti di questo tipo siano riscontrabili anche per altri elementi quando la compressione sia sufficientemente forte da provocare una sovrapposizione significativa delle distribuzioni elettroniche degli elettroni di cuore. Ad esempio, nel caso della struttura Na-hP4, la distanza $\mathrm{Na}-\mathrm{Na}$ più corta è pari a $1.89 \AA$, ovvero nettamente inferiore alla somma $(2.04 \AA)$ di due raggi ionici del Sodio. 


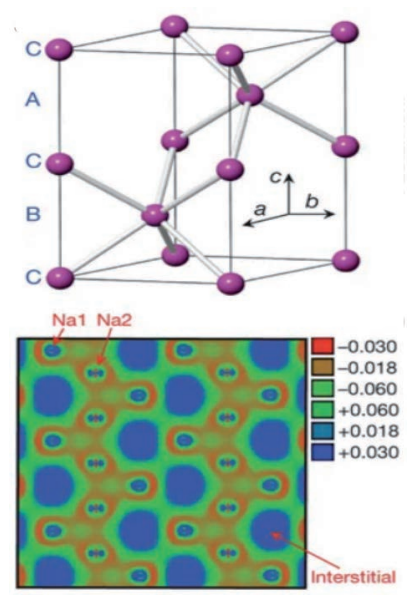

Fig. 10. Sopra: struttura cristallina di Na-hP4 (gruppo spaziale $\mathrm{P}_{3} / \mathrm{mmc}$ ) con due posizioni atomiche non equivalenti $(\mathrm{Na} 1$ a $2 a(0.0,0.0,0.0)$ e Na2 a $2 d(2 / 3,1 / 3,1 / 4)$; Sotto: densità elettronica differenza in e/ $\AA^{3}$ (densità elettronica nel cristallo meno la sovrapposizione delle densità degli atomi isolati disposti nelle stesse posizioni degli atomi del cristallo) nel piano (110) a $320 \mathrm{GPa}$. Si noti la presenza di significativi massimi della densità elettronica nelle posizioni interstiziali. Adattata da Fig. 4 del Rif. [22].

\subsection{Inusuali stechiometrie: $\mathrm{Na} a_{x} \mathrm{Cl}(x=1.33,1.5,2,3)$ e $\mathrm{NaCl} y(y=3,7)$}

Siamo abituati a vedere il nostro sale da cucina, il salgemma, $\mathrm{NaCl}$, come qualcosa di indefinitamente stabile, a meno che non lo si sciolga mettendolo in un solvente polare, come l'acqua. Riposto negli scaffali di cucina, sappiamo che è igroscopico e che quindi va protetto da un'eccessiva umidità dell'ambiente. Ma sicuramente riteniamo la sua stechiometria, cioè il rapporto 1:1 tra atomi di sodio ed atomi di cloro, qualcosa di perfettamente assodato e immutabile. Studi recenti, riportati su Science e su altre riviste [23-24] hanno invece dimostrato che a pressioni maggiori di $20 \mathrm{GPa}, \mathrm{NaCl}$ diventa reattivo rispetto ai suoi stessi componenti formando composti termodinamicamente stabili, arricchiti in $\mathrm{Na}$ o in $\mathrm{Cl}$ rispetto a $\mathrm{NaCl}$ e di formule generali $\mathrm{Na}_{\mathrm{x}} \mathrm{Cl}$ $(\mathrm{x}=1.33,1.5,2,3)$ e $\mathrm{NaCl}_{\mathrm{y}}(\mathrm{y}=3,7)$. Si tratta di composti con stechiometrie del tutto estranee alle regole elementari della chimica e che se fossero riportate su un elaborato di un esame scritto o alla lavagna durante un esame orale assicurerebbero all'improvvido studente un'istantanea, solenne bocciatura, sia in una scuola superiore, sia all'università. 
Eppure ad alta pressione questi composti corrispondono a fasi termodinamicamente stabili, con entalpie di formazione dagli elementi o da ogni altro composto ipotizzabile invariabilmente negative. Particolarmente sorprendenti appaiono le strutture ricche in sodio, $\mathrm{Na}_{\mathrm{x}} \mathrm{Cl}$. Presentano quattro diverse stechiometrie e un grande numero, ben superiore a quattro, di strutture cristalline diverse. Ad esempio le due strutture di $\mathrm{Na}_{3} \mathrm{Cl}$ a più bassa pressione sono metalliche e costituite da strati alternati di $\mathrm{NaCl}$ e di puro sodio. I sottoreticoli di $\mathrm{Na}$ bidimensionale possono essere descritti come strati metallici carichi positivamente che interagiscono con anioni Cloro che acquisiscono cariche negative superiori a 1 , mentre gli atomi di Sodio risultano meno carichi positivamente di quanto lo siano nel salgemma in quanto fanno anche parte di reticoli bidimensionali e pseudo-metallici di Na. Altre strutture ricche in Sodio, a pressioni più elevate, o con diverse stechiometrie, presentano reticoli di $\mathrm{Na}$ monodimensionali o assenza di legami $\mathrm{Na}-\mathrm{Na}$.

\subsection{Superata l'ultima barriera dell'inerzia chimica: il caso del $\mathrm{Na}_{2} \mathrm{He}$}

L'Elio $(\mathrm{He})$ è il secondo elemento per abbondanza nell'universo dopo l'idrogeno ed è presente in quantità significative nelle stelle e nei pianeti giganti gassosi quali Giove e Saturno. [35] L'Elio e il Neon sono gli elementi più inerti della TP. Il fatto non è sorprendente se si considera che l'atomo di He ha il maggiore potenziale di ionizzazione $(24.59 \mathrm{eV})$ tra tutti gli elementi [36] e la sua affinità elettronica è nulla. [37] Malgrado ciò, recentemente molti scienziati hanno cercato di trovare dei composti stabili dell'He. Gli esempi più significativi sono il radicale $\mathrm{HeH}$ [38] ed in genere i radicali $\mathrm{He}_{n} \mathrm{H} \cdot(n=1-6)$ che sono estremamente aggressivi, in grado di protonare qualsiasi specie ma stabili solo nelle loro forme cariche positivamente $\left(\mathrm{HeH}^{+}, \mathrm{He}_{n} \mathrm{H}^{+}\right)$. Tutte le molecole neutre trovate per via sperimentale o teorica, quali $\mathrm{HHeF}$, $\mathrm{HeO}, \mathrm{CsF}$ e LiHe sono risultate invariabilmente metastabili e molto ricche da un punto di vista energetico. Ad esempio $\mathrm{HHeF}$ ha un'energia di $2 \mathrm{eV}$ per atomo più alta di quella di una miscela 1:1 di molecole di HF e atomi di He. [39] Gli unici sistemi stabili dell'He, conosciuti fino alla recentissima scoperta di $\mathrm{Na}_{2} \mathrm{He}$, [25] erano un solido di van der Waals, $\mathrm{NeHe}_{2}$, con entalpia di formazione prossima a zero [40] ed un complesso, $\left(\mathrm{H}_{2} \mathrm{O}\right)_{2} \mathrm{He}$, che diviene marginalmente stabile solo ad altissima pressione (>300 $\mathrm{GPa})$ [41] e che può essere meglio descritto 
come un composto di inclusione, $\mathrm{He} @ \mathrm{H}_{2} \mathrm{O}$, più che come vero composto chimico.

$\mathrm{Na}_{2} \mathrm{He}$ è del tutto diverso. [25] Risulta un composto solido stabile $(10-15 \mathrm{Kcal} / \mathrm{mole})$ già a pressioni relativamente piccole $(>113 \mathrm{GPa})$ ed ha una struttura, tipo fluorite, che può essere vista come una scacchiera tridimensionale (Fig. 11). I cubetti che la compongono hanno ai loro vertici nuclei di Sodio e sono occupati, in modo alterno, da atomi di He e da elettroni di valenza interstiziali sottratti agli atomi di $\mathrm{Na}$. In pratica, sotto pressione, la presenza degli atomi di He induce una localizzazione degli elettroni di valenza del $\mathrm{Na}$ in posizione interstiziale, rendendo il materiale un isolante. Analogamente al sodio $\mathrm{Na}-\mathrm{hP} 4$ di cui abbiamo parlato in precedenza, $\mathrm{Na}_{2} \mathrm{He}$ è un elettruro con coppie elettroniche localizzate che assumono il ruolo di anioni doppiamente carichi (-2) e che formano legami a 8 centri e 2 elettroni (8c-2e) all'interno dei cubi di $\mathrm{Na}_{8}$ vuoti. Ogni coppia di elettroni interstiziali (e ogni atomo di $\mathrm{He}$ ) risulta coordinata/o da 8 atomi di Sodio. Nei classici elettruri dei sistemi a bassa pressione gli elettroni localizzati in posizioni interstiziali sono non accoppiati e pertanto polarizzati per spin, mentre in $\mathrm{Na}_{2} \mathrm{He}$ e $\mathrm{Na}-\mathrm{hP} 4$ gli elettroni interstiziali viaggiano in coppia. Sono un esempio di una classe del tutto nuova di elettruri che diviene stabile ad alta pressione e che forma composti altamente isolanti in tutto il loro campo di stabilità.

Uno dei lati più interessanti di questo studio è l'aver predetto il primo vero composto stabile di un elemento finora considerato del tutto inerte, attraverso conti quantomeccanici da principi primi e l'uso di algoritmi di selezione delle strutture energeticamente più stabili mutuati dai meccanismi che regolano l'evoluzione genetica. [33] E tutto ciò si è realizzato grazie ad una ricerca sistematica, [25] impossibile da effettuare in modo così esteso per via sperimentale, di tutte le possibili combinazioni tra He e gli altri elementi della TP. D'altra parte, utilizzando metodi di sintesi chimica e metodi di indagine cristallografici si è poi potuto verificare la validità della predizione teorica. [25]

Concludo questo paragrafo con gli incisivi commenti di Mitch Jacoby, "Chemistry textbook authors may soon have to rewrite sections covering noble gases and chemical inertness" e del Prof. Sven Lidin, membro della Royal Swedish Academy of Sciences, "The last bastion of chemical inertness has finally fallen", entrambi pubblicati in un editoriale di Chemical Engineering News dedicato a questo lavoro. [26] 


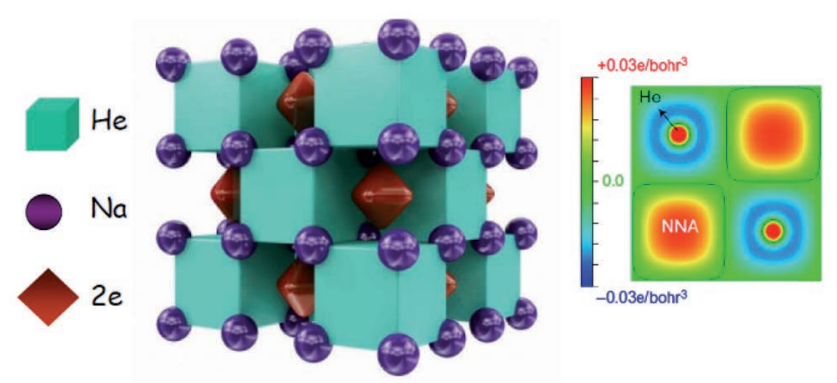

Fig. 11. A sinistra: Struttura geometrica ed elettronica a scacchiera tridimensionale di $\mathrm{Na}_{2} \mathrm{He}$ (adattata da Fig. 1 del Rif. [26] con permesso da e credito a Artem Oganov, Skolkovo Institute, Moscow, Russia; $A$ destra: Densità elettronica (e/bohr $\left.{ }^{3}\right)$ di interazione di $\mathrm{Na}_{2} \mathrm{He}$ nel piano (100) passante attraverso gli atomi di He e le coppie elettroniche interstiziali. La densità di interazione è stata calcolata come differenza tra la densità elettronica nel cristallo di $\mathrm{Na}_{2} \mathrm{He}$ e quella nei sottoreticoli di $\mathrm{Na}$ e di He presi separatamente, ovvero nei reticoli $\mathrm{Na}_{2} \mathrm{He}$ privati, rispettivamente, degli atomi di $\mathrm{He}$ o degli atomi di Na. Il simbolo NNA (Non-Nuclear Attractor) indica la localizzazione interstiziale di coppie elettroniche, causata dalla presenza di He che sposta la densità elettronica di valenza del Na negli spazi interstiziali. Adattata dalla Fig. 4 del riferimento [25].

\section{Note CONCLUSIVE}

In quest'esposizione sugli effetti della pressione sul comportamento dei composti chimici e su come questi modifichino il concetto di periodicità in chimica, ho volutamente privilegiato gli aspetti di natura più fondamentale. Ciò non vuol dire, come ho fatto intravedere in alcune parti della mia relazione, che gli effetti della pressione non abbiano risvolti pratici e tecnologici estremamente interessanti, la cui importanza è solo destinata ad accrescersi negli anni a venire. Ne è conferma uno sguardo a quello che è forse il libro più recente ed esauriente sul tema e che è significativamente intitolato An introduction to High Pressure and Technology. [43] Dei sedici capitoli che lo compongono, i primi dieci sono dedicati agli aspetti di base dell'alta pressione, ma ben sei ne delineano le applicazioni tecnologiche. Di questi, un primo capitolo tratta della microbiologia sotto pressione ed un secondo degli effetti della pressione sui principali composti alimentari, con un focus per entrambi sugli sviluppi tecnologici per la conservazione degli alimenti. Un terzo capitolo delinea le applicazioni della pressione nel campo delle biotecnologie e dei settori medico e farmaceutico. Un capitolo è 
poi interamente dedicato all'uso delle alte pressioni e delle alte temperature nella sintesi di sistemi inorganici. Si pensi a tal proposito all'importanza delle sintesi idrotermali nella genesi dei minerali terrestri o, in generale, allo sviluppo crescente delle sintesi solvotermali, in particolare nel campo della sintesi di nano-materiali. Infine, gli ultimi due capitoli riguardano temi di geofisica e astrobiologia. La pressione ha effetti profondi sulla composizione interna della terra e sulle proprietà che ne derivano, con grosse implicazioni sul comportamento sismico terrestre, mentre lo studio delle lune ghiacciate, di cui sono un esempio i satelliti galileiani di Giove, ha un interesse legato alla possibilità di vita nelle ampie riserve d'acqua del loro interno, a pressioni relativamente accettabili, dell'ordine dei 0.02-1 GPa. Si noti che il tema dell'abitabilità delle lune ghiacciate è una delle maggiori priorità nelle roadmaps della ricerca in campo spaziale. Ad esempio, l'European Space Agency (ESA) ha in programma per il 2022 il lancio della missione JUICE (JUpiter and ICy moons Explorer). Nell'attesa dei dati che verranno via via disponibili attraverso queste missioni, gli studi e gli esperimenti ad alte pressioni sui sistemi ghiacciati e in soluzione, non solo acquosi e acquose, sono di grande importanza per acquisire conoscenze sugli ambienti interni e nascosti di questi interessantissimi corpi planetari, a noi relativamente vicini. [44]

\section{BIBLIOGRAFIA}

1. Stilo G, Il sogno del chimico, Query https://www.queryonline.it/2019/03/15/ilsogno-del-chimico/, 15 marzo 2019

2. Gabici F, Chimica: i 150 anni della tavola periodica di Mendeleev, il rivoluzionario degli elementi, Avvenire https:/www.avvenire.it/agora/pagine/mendeleev-tavolaperiodica-degli-elementi-150-anni, 27 febbraio 2019

3. Mendeleev DI, On the Correlation Between the Properties of Elements and Their Atomic Weight, Zurnal Russkogo Kimicheskogo Obshchestva, 1869: 1: 60-77.

4. Mendeleev DI, A natural system of the elements and its use in predicting the properties of undiscovered elements, Zhurnal Russkoe Fiziko-Khimicheskoe Obshchestvo, 1871: 3: 25-56.

5. Schettino V, La materia in condizioni estreme. Una Chimica dell'altro mondo, $L a$ Chimica e l'Industria web, 2014: 2; 43-55.

6. Grochala V, Hoffmann R, Feng J, Ashcroft NW, The Chemical Imagination at Work in Very Tight Places, Angew. Chem. Int. Ed., 2007: 46: 3620-3642.

7. Ashcroft NW, Mermin ND, Solid State Physics, W.B. Saunders Company, International Edition, Saunders College, Philadelphia, 1976. 
8. Chalmers M, Pressure turns simplicity into complexity, ESRFnews, 2012 December.

9. Euchems (European Chemical Society) Periodic Table: 90 elementi chimici e la loro disponibilità sulla terra. Ci basteranno? https://www.euchems.eu/euchems-periodic-table/, 2019

10. Chalmers M, Modern-day alchemy is putting the periodic table under pressure, New Scientist, https://www.newscientist.com/article/mg22730380-500-modernday-alchemy-is-putting-the-periodic-table-under-pressure/[06/03/2019 09:56:33], 2019.

11. McMillan PF, New materials from high-pressure experiments, Nature Mat., 2002: 1: $19-25$.

12. Hazen RM, The diamond makers, Cambridge University Press, Cambridge, 1999.

13. Bridgman PW, The coaugulation of albumen by pressure, J. Biol. Chem., 1914: 19: 511-512.

14. Saldo J, Microbiology under pressure: how microorganisms are affected by pressure and how they may cope with it, in An introduction to High Pressure and Technology, Recio JM, Menéndez JM, Otero de la Roza A editori, 332-349, CRC Press, Taylor \& Francis group, Boca Raton, Florida, US, 2015.

15. Guignon B, Food Science and Technology: preservation and processing below 50 $\mathrm{MPa}$ and up to $1 \mathrm{GPa}$, in An introduction to High Pressure and Technology, Recio JM, Menéndez JM, Otero de la Roza A editori, 350-370, CRC Press, Taylor \& Francis group, Boca Raton, Florida, US, 2015.

16. Jayaraman A, Diamond anvil cell and high-pressure physical investigations, Rev. Mod. Phys. $1983: 55: 65-108$.

17. Baonza VG, Sánchez-Benítez J, High-Pressure Generation and Pressure Scales, in An introduction to High Pressure and Technology, Recio JM, Menéndez JM, Otero de la Roza A editori, 332-349, CRC Press, Taylor \& Francis group, Boca Raton, Florida, US, 2015.

18. https://en.wikipedia.org/wiki/Diamond_anvil_cell

19. Tse JS, Boldyreva EV, Electron Density Topology of Crystalline Solids at High Pressure, in Modern Charge-Density Analysis, Gatti C and Macchi P editori, 573. 623, Springer, Dordrecht, 2012.

20. Oganov AR, Chen J, Gatti C, Ma YZ, Ma YM, Glass CW, Liu Z, Yu T, Kurakevych OO, Solozhenko VL, Ionic high-pressure form of elemental boron, Nature 2009: 457: 863-868.

21. Oganov AR, Solozhenko VL, Gatti C, Kurakevych OO, Godec YL, The high-pressure phase of boron, $\gamma$-B28: disputes and conclusions of 5 years after discovery, $J$. Superbard Materials, 2011: 33:363-379.

22. Ma YM, Eremets M, Oganov AR, Xie Y, Trojan I, Medvedev S, Lyakhov AO, Valle M, Prakapenka V, Transparent Dense Sodium, Nature 2009: 458: 182-185.

23. Zhang W, Oganov AR, Goncharov AF, Zhu Q, Boulfelfel SE, Lyakhov AO, Stavrou E, Somayazulu M, Prakapenka VB, Konôpková Z, Unexpected Stable Stoichiometries of Sodium Chlorides, Science, 2013: 342: 1502-1505.

24. Saleh G, Oganov AR, Alkali subhalides: high-pressure stability and interplay between metallic and ionic bonds, Phys. Chem. Chem. Phys., 2016: 18: 2840-2849. 
25. Dong X, Oganov AR, Goncharov AF, Stavrou E, Lobanov S, Saleh G, Qian G.-R, Zhu Q, Gatti C, Deringer VL, Dronskowski R, Zhou X.-F., Prakapenka VB, Konôpková Z, Popov I, Boldyrev AI, Wang H-T, Stable Compound of Helium and Sodium at High Pressure, Nature Chemistry, 2017: $9: 440-445$.

26. Struzhkin VV, Hemley RJ, Mao H.-k., Timofeev YA, Superconductivity at 10-17 K in compressed sulphur, Nature $1997: 390: 382-384$.

27. Mailhiot C, Yang LH, McMahan AK, Polymeric nitrogen Phys. Rev. B, 1992: 46: 14419-14435.

28. Eremets MI, Gavriliuk AG, Trojan IA, Single-crystalline polymeric nitrogen, Appl. Phys. Lett., 2007: 90: 171904.

29. Petryanov-Sokolov IV, Popular Library of the Elements, Nauka, Russia, 1983.

30. Douglas BE, HO, S-M, Structure and Chemistry of Crystalline Solids, Springer, Dordrecht, 2006.

31. Chase MW Jr, NIST-JANAF Thermochemical Tables, Part I and Part II, J. Phys. Chem. Ref. Data 1998: Monograph 9: 1-1993.

32. Wohler F, Elements de Chimie Inorganique Et Organique, Kessinger Publishing, ristampa e traduzione in francese nel 2010 del libro originario del 1858.

33. Oganov AR, Ma Y, Lyakhov AO, Valle M, Gatti C, Evolutionary crystal structure prediction as a method for the discovery of minerals and materials, Rev. Mineral Geochem. 2010: 71: 271-298.

34. Celestino T, Lo strano caso dell'elemento Boro, Urto efficace (blog), 2009: 1 febbraio.

35. Stevenson, DJ, Metallic helium in massive planets, Proc. Natl Acad. Sci. USA, 2008: 105: 11035-11036.

36. Huheey JE, Keiter EA, Keiter RL, Inorganic Chemistry: Principles of Structure and Reactivity, HarperCollins College Publishers, New York, US, 1993.

37. Hotop H, Lineberger WC, Binding energies in atomic negative ions: II, J. Phys. Chem. Ref. Data, 1985: 14: 731-750.

38. Hiby JW, Massenspektrographische untersuchungen an wasserstoff- und heliumkanalstrahlen $\left(\mathrm{H}_{3}{ }^{+}, \mathrm{H}_{2}^{-}, \mathrm{HeH}^{+}, \mathrm{HeD}^{+}, \mathrm{He}^{-}\right)$. Annalen der Physik, 1939: 426: 473-487.

39. Wong MW, Prediction of a metastable helium compound: HHeF, J. Am. Chem. Soc., 2000: 122: 6289-6290.

40. Loubeyre P, Jean-Louis M, LeToullec R, Charon-Gérard L, High pressure measurements of the He-Ne binary phase diagram at $296 \mathrm{~K}$ : evidence for the stability of a stoichiometric $\mathrm{Ne}(\mathrm{He})_{2}$ solid, Phys. Rev. Lett., 1993: 70: 178-181.

41. Liu H, Yao Y, Klug DD, Stable structures of $\mathrm{He}$ and $\mathrm{H}_{2} \mathrm{O}$ at high pressure, Phys. Rev. B 2015: 91: 014102.

42. Jacoby M, Helium forms stable molecules at high pressures. Findings broaden understanding of chemical reactivity, inertness, and astrochemistry, Chemical $\mathcal{E}$ Engineering News, 2017: 95: 5-6.

43. Recio JM, Menéndez JM, Otero de la Roza A, Saldo J, An introduction to High Pressure and Technology, CRC Press, Taylor \& Francis group, Boca Raton, Florida, US, 2015. 
44. Prieto-Ballesteros O, Muñoz-Iglesias V, Bonales LJ, Interiors of Icy Moons from an Astrobiology Perspective: Deep Oceans and Icy Crusts, in An introduction to High Pressure and Technology, Recio JM, Menéndez JM, Otero de la Roza A editori, 453 481, CRC Press, Taylor \& Francis group, Boca Raton, Florida, US, 2015. 